\title{
Atividade Científica num Núcleo de Internato de Medicina Geral e Familiar
}

André F. Correia*, CDC.MGF**

Pontos-chave:

A produção científica e a participação ativa em reuniões clínicas e formativas são parte integrante e fundamental do processo de aprendizagem em Medicina Geral e Familiar.

Este estudo caracteriza a atividade científica e formativa dinamizada por um grupo de médicos de família num núcleo de internato, quer quanto ao tipo de atividade, quer quanto à área clínica classificada segundo a ICPC-2, procurando ainda refletir e partilhar a sua experiência com os restantes colegas e núcleos de formação do país.

\section{Resumo}

Introdução: Em Medicina Geral e Familiar (MGF) a produção científica e a formação contínua são extremamente importantes, especialmente na fase de pós-graduação. Em 2011, um grupo de internos de MGF de uma sub-região de Portugal decidiu criar um grupo de trabalho (CDC.MGF) com o objetivo de organizar as reuniões mensais dessa sub-região, promovendo a apresentação regular de trabalhos técnico-científicos, quer pelos próprios (ou orientadores) quer por médicos convidados de outras especialidades. O grupo desenvolveu ainda atividades formativas, como cursos e workshops.

Objetivo e Métodos: Caracterizar toda a atividade dinamizada pelo grupo desde a sua criação, classificando-a nas diversas tipologias de atividade científica e nas diversas áreas clinicas segundo a classificação ICPC-2.

Resultados: Desde 2011 o grupo promoveu um total de 94 iniciativas e apresentações científicas, das quais 67 (71,3\%) foram realizadas por MF em pós-graduação e 27 $(28,7 \%)$ por convidados de outras especialidades. Distribuíram-se nas seguintes tipologias: 48 (51,1\%) trabalhos de revisão, 15 (16\%) guidelines nacionais, 13 (13,8\%) casos clínicos, 5 (5,3\%) trabalhos de melhoria contínua da qualidade, 4 (4,3\%) trabalhos de investigação, 2 $(2,1 \%)$ trabalhos relacionados com a prática médica e registos clínicos e 7 (7,4\%) atividades de formação, nomeadamente 5 cursos e 2 workshops. Quanto à classificação ICPC-2, as áreas mais abordadas foram: A (geral e inespecífico) em 17,6\%; T (endócrino, metabólico e nutricional) em 14,7\%; L (sistema musculoesquelético) e R (aparelho respiratório) em 11,8\% cada; e P (psicológico) em 9,8\%. Destes trabalhos, 19 foram dedicados exclusivamente a crianças e adolescentes, 4 a saúde materna e gravidez e 3 a idosos.

Discussão/Conclusão: Estes resultados mostram que este grupo apresenta uma atividade diversificada quer por tipologias quer por áreas científicas, com uma participação significativa de médicos de outras especialidades. Encontra-se um predomínio dos trabalhos de revisão, refletindo a sua importância na atualização contínua dos profissionais. No futuro o grupo deverá incentivar atividades em outras áreas menos abordadas, promovendo uma abordagem holística, característica fundamental em MGF.

MeSH: "family practice”; “continuing education”; “internship and residency"; "primary health care/classification”

\footnotetext{
* Interno de Formação Específica em Medicina Geral e Familiar, UCSP Águeda V, ACeS Baixo Vouga

** Comissão Dinamizadora de Conteúdos em Medicina Geral e Familiar
} 


\section{Introdução}

A Medicina Geral e Familiar (MGF) constitui atualmente uma das áreas médicas mais abrangentes e mais reconhecidas a nível nacional e internacional. Define-se enquanto disciplina académica - com uma prática, ensino, investigação e avaliação da qualidade próprias - e enquanto atividade profissional, isto é, enquanto especialidade que aplica na prática clínica em cuidados de saúde primários as características e competências da disciplina, indo de encontro às necessidades de saúde das populações ${ }^{1}$. A sua evolução tem motivado uma reformulação periódica dos programas de formação pré e pós-graduada ${ }^{2,3}$ e uma atualização contínua dos conhecimentos e aptidões dos profissionais (internos e especialistas), visando assim uma prestação mais segura e efetiva dos cuidados de saúde nas populações.

O programa de formação específica pós-graduada em MGF em Portugal prevê que o interno possa desenvolver a sua prática clínica e integrar conhecimentos, aptidões e atitudes, capacitando-se assim para exercer de forma autónoma e competente no final do internato. ${ }^{4}$ Além da observação e treino no local de trabalho, o internato em MGF deve incentivar outras estratégias de aprendizagem, como a participação em cursos, a elaboração de trabalhos científicos e a dinamização de sessões clínicas e formativas com outros profissionais. ${ }^{5}$

É neste contexto que têm surgido um pouco por todo o país vários agrupamentos de médicos de família (MF), constituídos maioritariamente por internos, que procuram promover uma maior proximidade e colaboração entre colegas e a dinamização dos seus núcleos de internato. Fazem-no incentivando a partilha de conhecimentos, a discussão de assuntos relevantes à formação e a organização de cursos e outros eventos científicos e educativos, de forma autónoma ou em parceria com as Direções de Núcleos Locais e com as Coordenações Regionais de Internato.

Em 2011, vários internos do núcleo de internato da sub-região de Aveiro (que abrangia então os agrupamentos de centros de saúde - ACeS - Baixo Vouga I, II e III, atualmente fundidos no ACeS Baixo Vouga), decidiram criar um grupo de trabalho com vista a organizar e sistematizar, conjuntamente com a direção local do internato, as reuniões mensais entre internos e orientadores da região. Pretendeu-se assim promover a apresentação regular de trabalhos científicos por internos e especialistas de MGF e de sessões clínicas com médicos convidados de outras especialidades.
Este grupo, denominado CDC.MGF (Comissão Dinamizadora de Conteúdos em Medicina Geral e Familiar), tem promovido ainda a realização de outras atividades formativas, como cursos e workshops em várias áreas (respiratória, diabetologia, psiquiatria, saúde sexual e reprodutiva nas escolas, análise estatística, entre outras), e mais recentemente o seu primeiro evento científico, a Academia Médica, em 2014.

Os autores deste artigo procuraram assim estudar as atividades dinamizadas pelo grupo CDC.MGF entre $2011 \mathrm{e}$ 2013, de modo a inferir quais as áreas mais abordadas e gerar discussão sobre atividades alternativas e temas menos explorados.

\section{Metodologia}

Estudo descritivo, transversal e retrospetivo, realizado em dezembro de 2013.

Foram consultadas as ordens de trabalhos e respetivos resumos (quando existentes) das reuniões de núcleo de internato de MGF de Aveiro/ACeS Baixo Vouga desde a criação do grupo CDC.MGF em 2011 até dezembro de 2013. Foram igualmente listados os diferentes cursos de formação e workshops organizados pelo grupo.

As apresentações e formações realizadas foram classificadas de acordo com: 1) tipo de atividade científica; 2) área clínica, segundo o sistema de classificação internacional de cuidados primários ICPC-2 (podendo existir mais que um campo por atividade).

Foram consideradas como atividades científicas: revisão de tema; guidelines nacionais/Normas de Orientação Clínica (NOC) da Direção Geral de Saúde (DGS); relato de caso; melhoria contínua da qualidade; investigação; trabalhos relacionados com a prática médica e registos clínicos; atividades de formação (cursos e workshops).

Foram ainda analisadas as atividades dedicadas em exclusivo a um determinado grupo populacional (infantojuvenil, grávidas e idosos).

0 registo e o tratamento estatístico dos dados foram realizados através do programa Microsoft Excel. 


\section{Resultados}

Entre 2011 e 2013, o grupo promoveu um total de 94 iniciativas e apresentações científicas, a maioria das quais realizadas por internos de MGF (71,3\%). De entre essas iniciativas, cerca de metade $(51,1 \%)$ foram trabalhos de revisão, seguindo-se as apresentações de guidelines nacionais e de casos clínicos. Trabalhos de melhoria contínua da qualidade, de investigação e relacionados com a prática médica foram apresentados em menor número. Realizaram-se ainda 7 atividades de formação, nomeadamente 5 cursos e 2 workshops.

Tabela 1: Descrição das atividades científicas por especialidade médica dos autores/formadores e por tipologia científica

\begin{tabular}{lc|c} 
Variável & N $^{\circ}$ & $\%$ \\
\hline \multicolumn{2}{l|}{ Especialidade médica dos autores/formadores } & \\
\hline Internos de MGF & 67 & 71,3 \\
Especialistas não-MF convidados & 27 & 28,7 \\
\hline Atividades realizadas segundo tipologia científica & \\
\hline Cursos e workshops & 7 & 7,4 \\
Guidelines nacionais / NOC & 15 & 16,0 \\
Investigação & 4 & 4,3 \\
Melhoria contínua da qualidade & 5 & 5,3 \\
Prática médica e registos clínicos & 2 & 2,1 \\
Relato de caso & 13 & 16,0 \\
Revisão de tema & 48 & 51,1
\end{tabular}

Quanto à classificação ICPC-2, as 94 atividades foram catalogadas em 102 áreas clínicas. As cinco áreas (capítulos) mais abordadas foram: A (geral e inespecífico), T (endócrino, metabólico e nutricional), L (sistema musculoesquelético), R (aparelho respiratório) e $\mathrm{P}$ (psicológico). Estas compõem quase dois terços das atividades realizadas. Por sua vez, as áreas hematológica (B), digestiva $(C)$, otológica $(H)$, social $(Z)$, oftalmológica (E), dermatológica (S) e genital masculina (Y) foram abordadas pontualmente.

Tabela 2: Distribuição das atividades dinamizadas segundo a Classificação Internacional para Cuidados Primários (ICPC-2)

\begin{tabular}{|c|c|c|c|}
\hline & Capítulos ICPC-2 & $\mathbf{N}^{\circ}$ & $\%$ \\
\hline A & Geral e inespecífico & 18 & 17,6 \\
\hline B & $\begin{array}{l}\text { Sangue, órgãos hematopoiéticos e } \\
\text { linfáticos }\end{array}$ & 1 & 1,0 \\
\hline D & Aparelho digestivo & 1 & 1,0 \\
\hline $\mathrm{F}$ & Olhos & 2 & 2,0 \\
\hline $\mathrm{H}$ & Ouvidos & 1 & 1,0 \\
\hline K & Aparelho circulatório & 7 & 6,9 \\
\hline L & Sistema musculoesquelético & 12 & 11,8 \\
\hline N & Sistema nervoso & 5 & 4,9 \\
\hline P & Psicológico & 10 & 9,8 \\
\hline R & Aparelho respiratório & 12 & 11,8 \\
\hline S & Pele & 2 & 2,0 \\
\hline T & Endócrino, metabólico e nutricional & 15 & 14,7 \\
\hline U & Aparelho urinário & 4 & 3,9 \\
\hline W & Gravidez e planeamento familiar & 4 & 3,9 \\
\hline$x$ & Aparelho genital feminino e mama & 5 & 4,9 \\
\hline Y & Aparelho genital masculino & 2 & 2,0 \\
\hline \multirow[t]{2}{*}{ Z } & Problemas sociais & 1 & 1,0 \\
\hline & TOTAL & 102 & 100,0 \\
\hline
\end{tabular}

Quanto às atividades dedicadas em exclusivo a um dado grupo populacional, 19 (20,2\%) foram dedicados a crianças e adolescentes, 4 (4,3\%) a saúde materna e gravidez e $3(3,2 \%)$ a idosos.

\section{Discussão}

O internato em MGF em Portugal, apesar de recente (iniciado na década de 80 enquanto Clínica Geral, consolidado na década de 90 e reformulado ao longo dos anos) $)^{6}$, estabeleceu precocemente os seus requisitos de formação, sendo o seu programa formativo considerado atualmente como um dos mais organizados e de maior qualidade no panorama da formação pós-graduada. ${ }^{7}$

Os encontros entre internos, orientadores de MGF e profissionais de outras áreas/especialidades clínicas, promovidos em reuniões, sessões clínicas ou eventos formativos, fomentam a comunicação e colaboração interpessoal, a discussão e a partilha de competências e metodologias, contribuindo para a evolução dos participantes e da própria MGF. 
No caso concreto das reuniões deste núcleo, além do espaço de discussão de assuntos relacionados com o internato, estas incluem regularmente uma sessão dinamizada por médicos de outras especialidades, uma NOC da DGS e uma a duas apresentações dinamizadas por Internos ou especialistas de MGF locais.

Não é comum a publicação de artigos em revistas nacionais da especialidade que relatem e descrevam a atividade dinamizada em reuniões e cursos nos núcleos locais de internato, embora seja de admitir a existência de trabalhos similares apresentados em jornadas científicas e congressos, como ocorreu recentemente na $19^{a}$ conferência da WONCA Europe em Lisboa. ${ }^{8,9}$

Será ainda menos comum, para não dizer pioneira, a descrição das atividades segundo a tipologia científica e segundo a ICPC-2. Relativamente a esta última classificação, esta foi recentemente proposta e utilizada como parte de um sistema de classificação das comunicações selecionadas e apresentadas em jornadas e congressos. ${ }^{10}$

Relativamente aos resultados obtidos, verifica-se um predomínio dos trabalhos de revisão. Tal dever-se-á, por um lado, à necessidade dos internos que os realizaram em esclarecer dúvidas ou consolidar conhecimentos nas áreas revistas, e por outro lado, ao tipo de apresentação que é geralmente promovida pelo profissional não-MF, convidado para cada reunião. Em segundo lugar encontram-se as NOC, apresentadas de forma curta e sucinta, num formato designado de "NOC em 5 minutos" que teve uma adesão mais sistemática neste último ano. São menos frequentes as atividades relacionadas com a prática clínica, melhoria de qualidade e investigação. Se relativamente às primeiras se pode considerar uma frequência aceitável e expetável, em relação às restantes seria de esperar uma frequência maior, atendendo à existência de cursos curriculares obrigatórios nestas áreas e à valorização curricular dos internos aquando da realização de trabalhos deste tipo. A maior morosidade na conclusão deste tipo de projetos ou as dificuldades que muitas vezes apresentam na sua elaboração poderão explicar a menor frequência de apresentações na área da melhoria de qualidade e de investigação. 0 número de cursos e workshops pode refletir a juventude do grupo e o tempo investido, geralmente fora do período laboral, na organização dessas atividades.

As atividades dinamizadas neste núcleo de internato abordaram áreas clínicas diversificadas, com predomínio das áreas gerais, metabólicas, musculoesqueléticas, respiratórias e da saúde mental, frequentemente trabalhadas na prática clínica, ainda que não faça parte deste "top 5" a área cardiovascular, que abrange patologias tão prevalentes e muitas vezes complexas. Os resultados obtidos classificados de acordo com a ICPC-2 poderão ser explicados pelo interesse dos internos em determinada área clínica, pela maior prevalência de patologia nessa mesma área e pelo maior incentivo e/ou exigência na realização de trabalhos aquando da passagem do interno pelos estágios hospitalares.

Quanto aos grupos populacionais explorados, verificou-se uma percentagem assinalável de atividades exclusivamente vocacionadas para crianças e adolescentes, cujos crescimento, desenvolvimento e patologias são complexos, quer na sua compreensão teórica, quer na sua abordagem prática. Apesar do envelhecimento progressivo da população, e por conseguinte das listas de utentes, e dos idosos serem um dos principais utilizadores dos cuidados de saúde ${ }^{11}$, este grupo foi de entre os estudados aquele que foi menos explorado. Deve contudo ter-se em conta que a maioria dos temas explorados referentes à saúde/doença de adultos pode ser aplicada aos idosos, ainda que seja de incentivar futuramente uma melhor abordagem das especificidades e complexidades dos indivíduos deste grupo etário. 0 mesmo incentivo e complexidade são aplicáveis à área da saúde materna, ainda que nesta se encontrem divulgados e implementados protocolos locais e nacionais de atuação e vigilância de gravidezes de baixo risco e de referenciação hospitalar ${ }^{12}$, o que a somar à diminuição do número de gravidezes/ano poderá justificar os resultados obtidos nesta área. Os autores decidiram estudar estes grupos vulneráveis (e não outros, como aqueles sujeitos a Planeamento Familiar ou Rastreio Oncológico) pela diferença etária entre eles - não discriminável totalmente pela ICPC-2 - e pela especificidade que estes grupos comportam.

Os resultados apresentados traduzem uma diversidade de atividades, fruto do dinamismo do grupo e da participação dos internos no núcleo de internato. Permitem ainda reconhecer tendências e preferências destes profissionais, isto porque as comunicações das reuniões são propostas voluntariamente e aceites na sua totalidade pelo grupo - não há lista de espera, obrigatoriedade de apresentação nas mesmas ou critérios de pré-seleção, ao contrário do que acontece em alguns núcleos do país ${ }^{13}$. Tal poderá significar também uma maior participação de alguns internos mais ativos, em detrimento de outros, podendo muitas vezes ficar "na gaveta" apresentações científicas igualmente pertinentes e de elevada qualidade. Embora se possa especular acerca das razões que motivam a (não) apresentação dessas comunicações, tal não foi objeto de estudo deste trabalho. 
Os cursos e convidados promovidos traduzem igualmente a preferência do grupo, a disponibilidade dos palestrantes/formadores e o interesse e necessidade dos internos.

Considerando os resultados deste estudo, já apresentados em reunião de internato, espera-se gerar a discussão acerca da pertinência dos temas mais abordados, continuar a dinamizar mais e melhores formações e incentivar novas actividades, dando destaque a temáticas menos abordadas (indicadores de desempenho, problemas sociais; "journal clubs", maior participação dos orientadores/especialistas MF e dos internos menos participativos, entre outras sugestões dadas).

\section{Conclusão}

O presente estudo permitiu conhecer de forma sistematizada a atividade produzida localmente por um núcleo de internato. Foram reconhecidas as suas virtudes, as atividades de maior interesse para os seus elementos e as áreas passíveis de ser mais exploradas futuramente por este.

\section{Referências Bibliográficas}

1. European Academy of Teachers in General Practice. A definição europeia de Medicina Geral e Familiar (Clínica Geral / Medicina Familiar).Versão reduzida. Euract; 2005.

2. Portaria n. ${ }^{\circ} 300 / 2009$ de 24 de Março. Programa de formação do internato médico da área profissional de especialização de medicina geral e familiar. Ministério da Saúde. Diário da República, 1. ${ }^{a}$ Série, n. ${ }^{\circ} 58$ de 24 de Março de 2009, 1853-1857.

3. Portaria n. ${ }^{\circ}$ 251/2011 de 24 de Junho. Ministério da Saúde. Diário da República, 1. ${ }^{a}$ série - N. ${ }^{\circ} 120$ - 24 de Junho de 2011.

4. Coordenação do Internato Complementar da Zona Norte. Caderneta de estágio 2011. Porto: CIMGFZN, 2011.

5. Avaliação Final do Internato Médico. Colégio de Medicina Geral e Familiar. 2012 (disponivel em http://www.ordemdosmedicos.pt/?lop=conteudo\&op=dd458505749b2941217d dd59394240e8\&id=92426b262d11b0ade77387cf8416e 153, acedido a 4-9-2014)

6. Sousa JC; Sardinha, AM; Perez y Sanchez J; Melo M; Ribas MJ. Os cuidados de saúde primários e a medicina geral e familiar em Portugal. Rev Port Saúde Pública 2001; 2: 63-74

7. Henriques J; Alexandra D. Medicina Geral e Familiar: Desafios Hoje e no Futuro. Acta Med Port 2014 Mai-Jun; 27(3):287-288

\section{Conflito de Interesses}

Os autores declaram não ter conflitos de interesse, além dos relacionados com a dinamização das atividades referidas neste artigo.
Em conclusão, a existência de núcleos de internato dinâmicos e participativos, onde os profissionais se organizam de forma pro-ativa, contribuem para fomentar o espírito crítico e a atualização contínua de especialistas de MGF, de modo a serem prestados cuidados de saúde de qualidade.

\section{Agradecimentos}

Os autores, na pessoa do seu primeiro autor, agradecem à direção e aos elementos do núcleo de internato de Aveiro (internos e orientadores) por participarem e colaborarem na sua dinamização, com destaque especial para os restantes elementos do Grupo CDC.MGF à data da realização deste trabalho pela disponibilidade e colaboração na elaboração do mesmo: Tiago Maricoto; José Antunes; Diogo Pereira; Joana Sequeira; Inês Silva; Eliana Bonifácio; Ana Pereira; Margarida Neto; Joana Bordalo; Cláudia Rainho; Sara Vidal; Ana Galante; Catarina Carvalho; Sara Santana; Catarina Sebe; Joana Caniço.

8. Rumor L; Alves VC; Barata AN; Baptista J; Gaiteiro L. Keeping Family Medicine Residents Together - The Experience of a Group Organized by and for FM Residents in Portugal. (Abstract P01566). In 19th WONCA Europe Conference, July 2 to 5, 2014. Lisbon, Portugal

9. Correia A; CDC.MGF Group. Scientific activity in a Post-Graduation GPs Group. (Abstract P01188). In 19th WONCA Europe Conference, July 2 to 5, 2014. Lisbon, Portugal

10. Jamoulle M. Using the International Classification for Primary Care (ICPC) and the Core Content Classification for General Practice (3CGP) to classify conference abstracts. Rev Port Med Geral Fam 2013; 29(5):337-338

11. Balsinha MC; Gonçalves-Pereira M. A avaliação do paciente idoso em Medicina Geral e Familiar: desafios e oportunidades. Rev Port Med Geral Fam 2014; 30(3): 196-201

12. Norma 37/2011 Exames Laboratoriais na Gravidez de Baixo Risco. Direcção-Geral de Saúde; 2011 (atualizada em 2013).

13. Programa de Formação, Ano 2014. Direcção do Internato Egas Moniz; 2014 (disponivel em http://www.docvadis. pt/diem-cimgfzn/page/apoio_ao_interno_mgf_core/ reuni_es_diem/reuni_es_mensais_diem.html; acedido a 5-9-2014)

Endereço para Correspondência:

acorreia2308@gmail.com

cdc.mgf@gmail.com 